\title{
Optimal management of desmoid fibromatosis
}

Hong Kong Med J 2018;24:316

DOI: $10.12809 / \mathrm{hkmj} 187250$

To the Editor-I read with great interest the recent report by $\mathrm{Ng}$ et $\mathrm{al}^{1}$ of mesenteric fibromatosis. I would like to take this opportunity to clarify the internationally accepted treatment approach for desmoid fibromatosis. In the concluding remarks, the authors have rightfully stated that "...surgical resection is usually indicated in large symptomatic cases of MF or in MF with complications" and “... decision for radiotherapy or systemic treatment... should be made after discussion with oncologists". I cannot help but notice that they give the readers the impression that surgery remains the treatment modality of choice before the consideration of systemic or hormonal treatments. The authors have also referenced an outdated version of the European consensus that, incidentally, was further updated in 2017. ${ }^{2}$ Evidence suggests that surgery should only be considered as an option if the morbidity from the procedure is limited, and medical therapy, be it with hormonal agents, non-steroidal anti-inflammatory drugs, cytotoxics, or targeted therapies, should also be considered as first-line treatments. More importantly, I would like to emphasise that surgical margins have been shown to inconsistently correlate with recurrence. ${ }^{3}$ The common consensus is that an $\mathrm{R} 0$ resection is not necessary if it is at the expense of significant morbidities or risk of mortality. Ultimately, a multidisciplinary team approach is necessary to ensure the best possible outcome for patients with this rare disease.

\section{Declaration}

As an editor of this journal, the author was not involved in the peer review process of this article. The author had full access to the data, contributed to the study, approved the final version for publication, and take responsibility for its accuracy and integrity.

\section{Herbert HF Loong *, FHKCP, FHKAM (Medicine)}

Department of Clinical Oncology, Prince of Wales Hospital, Shatin, Hong Kong

*Corresponding author: h_loong@clo.cuhk.edu.hk

\section{References}

1. Ng EP, Kwok SY, Lok KF, Chow MP, Lau PY. Mesenteric fibromatosis: a rare cause of peritonitis. Hong Kong Med J 2018;24:84-6.

2. Kasper B, Baumgarten C, Garcia J, et al. An update on the management of sporadic desmoid-type fibromatosis: a European Consensus Initiative between Sarcoma PAtients EuroNet (SPAEN) and European Organization for Research and Treatment of Cancer (EORTC)/Soft Tissue and Bone Sarcoma Group (STBSG). Ann Oncol 2017;28:2399-408.

3. Salas S, Dufresne A, Bui B, et al. Prognostic factors influencing progression-free survival determined from a series of sporadic desmoid tumors: a wait-and-see policy according to tumor presentation. J Clin Oncol 2011;29:3553-8. 